\title{
幼儿园生活活动中教师指导策略的实践研究
}

马娜娜

东营市垦利区康居幼儿园

DOI:10.32629/jief.v2i11.2507

[摘 要] 随着我国教育的不断变革, 幼儿教育成为了我国整体教育体系中非常重要一部分。通过采用科学合理的指导策略, 可以帮助幼儿形 成良好的学习习惯，培养起幼儿自身的行动力和自理能力，使得他们能够在幼儿教育阶段便获得更加有效的身心发展，为其今后的生活奠定 良好的基础，同时也为其今后的自我管理提供更加有效的帮助。本文针对我国幼儿园教育过程中教师的指导策略进行了有效的探究，为指导 策略今后有效的改善和优化提供了一定的意见和建议。

[关键词] 幼儿教育; 生活活动; 指导策略

中图分类号：G613 文献标识码：A

在幼儿园教育过程中, 采用更加有效的生活活动指导, 能够帮助幼 儿形成积极向上的人生观和价值观, 同时还能够更加有效地培养他们的 自理能力, 促进其生活独立性发展。因此, 如何更好的在幼儿园生活活 动中进行指导, 是幼儿教师必须要研究的一个问题, 也是我国今后幼儿 教育实现有效提升的关键之一。

\section{1 生活活动中开展良好指导的价值}

1.1 打造轻松高效的学习氛围

在幼儿园教育过程中, 如果能够在日常的生活活动中更好的进行学 习, 可以有效的改善传统教学方式的枯燥性, 打造出更加轻松高效的学 习环境和氛围。比如, 在教育儿童认识汉字时, 采用日常的教学方式, 在课堂上进行不断的朗诵, 只会增加学生的排斥性, 对于其学习会造成 一定的不良影响, 但如果能够在日常的生活过程中, 将其遇到的汉字及 时的讲解, 便可以打造出更加良好的环境, 创设出更加轻松的氛围, 也 可以提升学生学习的效率。

1.2 实现生活场景的教育意义

生活便是最好的教育环境, 良好的生活活动指导能够更好的发挥出 生活教育作用, 实现更为有意义的教育。生活教育不同于传统课堂教育, 其能够将教育与生活进行有效的结合, 通过更加科学的引导方式, 实现 幼儿教育更加稳定高效的提升, 开发幼儿的生活潜能, 为其今后的生活 和发展提供更好的基础。

1.3 提升幼儿的生活性和独立感

在日常的生活活动中, 对幼儿进行教育和引导, 可以提升幼儿本身 的生活性以及独立感, 使他们更加快速的融入生活之中, 为其生活发展 提供更大的帮助和支持。比如, 在日常的午饭过程中, 引导学生们更好 的使用筷子, 可以帮助学生掌握更加有效的生活技能, 实现其生活性的 有效培养。同时通过引导学生通过使用筷子, 自主的进行吃饭, 还能够 形成其良好的独立感，为其今后的发展提供更大的帮助和支持。

\section{2 生活活动中进行指导的有效方法}

2.1 结合儿童特征, 开发出具有教育意义的游戏

儿童本身具有非常明显的活泼好动的天性, 因此在日常的幼儿园教 育过程中, 想要更好的进行生活活动的引导, 实现更好的教育意义, 就 必须更加有效的结合儿童特征, 通过开发出更加丰富、具有教育意义的 游戏, 提升整个生活活动引导的趣味性, 以达到更好的教育目的。比如, 在日常的生活活动引导过程中, 教师们可以通过组织系鞋带比赛、筷子 夹花生米比赛以及叠被子大赛, 培养孩子们自主系鞋带、自主叠被子以 及使用筷子的能力, 以此来达到更好的教育效果。同时, 通过采用游戏
的方式, 还能够增强整个学习的趣味性, 吸引孩子的注意力, 更好发挥 他们的学习主动性。

\section{2 加强鼓励和引导}

在对幼儿进行教育的过程中, 一定要加强鼓励和引导, 通过更好鼓 励, 使得他们能够对生活活动产生良好的兴趣。教师们在日常的教研和 引导过程中, 一定要注重自身的态度和方法, 不能够过于的刻板和严厉, 这样会影响到儿童本身的兴趣。只有在日常的生活活动中添加更好的趣 味性引导, 才能够促进儿童更加积极快乐的生活和学习。比如, 在幼儿 进行学习的过程中, 教师们可以通过发放小红花的方式, 来鼓励表现较 好的幼儿, 引导其他幼儿更好的进行学习, 争取更多的小红花, 进而发 挥出学习的主动性和积极性, 真正实现教育目的。

\section{3 引导幼儿进行自我管理}

在生活活动中, 进行指导的首要目的就是要培养学生的独立性, 真 正的为幼儿今后的发展奠定良好的基础。因此, 教师们在日常的教学过 程中, 除了应用多种方法进行教育和引导之外, 还必须要进行更好的幼 儿自我管理引导, 减少对于幼儿生活活动中的刻板化规定, 尽量发挥出 幼儿自我管理的能力。比如, 在日常的教学和生活过程中, 可以引导幼 儿进行自我管理, 通过建立轮流督导的形式, 使得幼儿都能够当监督员, 监督其他幼儿是否能够进行有效的学习和生活, 以此来更好培养幼儿自 我管理能力。

\section{3 总结}

随着我国幼儿教育的不断发展, 整体的教育水平获得了有效的提升, 在当前的幼儿园教育过程中, 更加强调对于幼儿生活活动的引导, 以实 现更加良好的教学目的。在生活活动中进行有效的引导, 可以打造出更 加轻松高效的环境和氛围, 充分发挥出生活场景的教育作用, 实现儿童 生活性和独立感的有效培养。因此, 在今后的幼儿教育过程中, 一定要 结合幼儿的特征开发出更为有效的引导方式, 同时还应当加强鼓励, 促 进幼儿自我管理意识的有效发展。

\section{[参考文献]}

[1]朱艺婷. 浅谈如何将幼儿科学教育活动生活化 [J]. 才 智,2020(16):60.

[2]曹莉莉.幼儿园数学教育的生活化与游戏化浅谈 [J]. 才 智,2020(12):220.

[3]李黎燕.让幼儿体验科学探究的魅力 [J].课程教材教学研究(教育 研究),2020(Z3):76-78. 